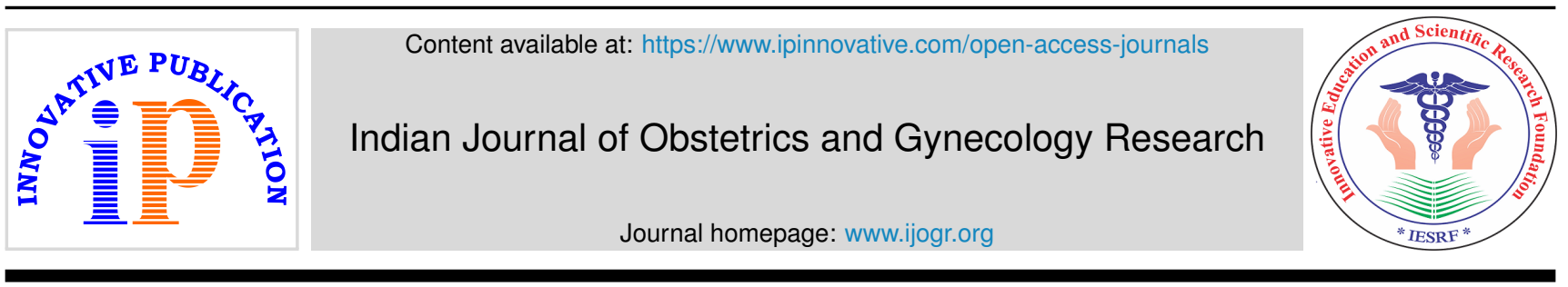

Original Research Article

\title{
Menstrual hygiene and their predictors among school going adolescent girls in Tamil Nadu
}

\author{
Lakshmi Subburaj $^{1, *}$, K Rajkumar ${ }^{1}$ \\ ${ }^{1}$ Dept. of Obstetrics and Gynecology, NTC Hospitals, Tamil Nadu, India
}

\section{A R T I C L E I N F O}

\section{Article history:}

Received 13-10-2020

Accepted 16-03-2021

Available online 11-06-2021

\section{Keywords:}

Adolescence

Dysmenorrhea

Menstrual hygiene

Reproductive tract infections

\begin{abstract}
A B S T R A C T
Background: The prevalence of menstrual problems is high during adolescence according to published literature. It is essential to determine the menstrual hygiene practices among the adolescent girls and their predictors so as to ascertain the modalities for prevention of menstrual problems. The present study was conducted to evaluate the menstrual problems, menstrual hygiene and their risk factors.

Materials and Methods: This cross-sectional study was carried out among 244 school going adolescent girls in the field practice area of our institution. The participants were selected using two stage sampling. A structured interview schedule was used to obtain information regarding the demographic details, menstrual problems and menstrual hygiene. The data was entered and analyzed using SPSS software. Chi square test was used to analyze the statistical significance.

Results: The present study showed a high prevalence for dysmenorrhea (75.4\%). About $72.1 \%$ of the participants consume over-the-counter medicines for menstrual problems. Majority of the participants (92.2\%) use sanitary napkins as absorbent materials. Only $22.1 \%$ of the participants wash their genitalia with soap and water during menstruation. About $10.2 \%$ of the participants do not take bath during menstruation. Age was a significant risk factor for menstrual hygiene $(\mathrm{p}<0.05)$.

Conclusion: The present study has emphasized on the need for creating awareness during the early adolescent age in order to inculcate hygienic menstrual practices. It is essential to make sanitary napkins available and affordable in the lower income groups. Moreover, there is a growing need to incorporate adolescent specific health services in the public health system.
\end{abstract}

(C) This is an open access article distributed under the terms of the Creative Commons Attribution License (https://creativecommons.org/licenses/by/4.0/) which permits unrestricted use, distribution, and reproduction in any medium, provided the original author and source are credited.

\section{Introduction}

The period of adolescence is characterized by pubertal changes and onset of menstruation. Being a crucial stage of physical, physiological and psychological transition from childhood to adulthood, the vulnerability towards various health issues and high-risk behaviors is higher at this stage. According to World Health Organization, globally, approximately 10 million unintended pregnancies occur among adolescent girls aged between 15-19 years each year. ${ }^{1}$ The high risk sexual exposures during the adolescent age group also significantly increases the risk of sexually transmitted and reproductive tract infections. Therefore, it

\footnotetext{
* Corresponding author.

E-mail address: lakmisubburaj@gmail.com (L. Subburaj).
}

is essential to provide adequate focus on the knowledge and practices related to menstruation, focusing on the adolescent age group, in order to ensure minimal exposure to these high-risk behavior and other reproductive health problems in their adult life.

Menstruation is a routine event in a woman's life. Throughout the reproductive life, women are often exposed to one or more of health risks associated with menstruation, namely dysmenorrhea, menorrhagia or irregular menstrual cycles. The prevalence of menstrual problems in a developing country like India is as high as $87.7 \%$ among the adolescent girls. ${ }^{2}$ Majority of these menstrual problems stems from lack of awareness towards menstruation and poor menstrual hygiene practices. Studies have shown that poor menstrual hygiene practices in terms 
of using unhygienic absorbent materials, poor hand washing practices were highly prevalent in India. ${ }^{3}$

Several risk factors have been attributed to these vulnerabilities among the adolescent age group. While these issues are highly prevalent among the lower socioeconomic groups, lack of awareness and inadequate access to health care services have been major causes. In addition, the scope for adolescent specific health services is significantly lower in emerging economies like India, especially in the public health infrastructure. This warrants the need to focus on adolescent specific issues and their risk factors. The present study was carried out to estimate the prevalence of menstrual problems and to assess the menstrual hygiene practices among the school going adolescent girls.

\section{Materials and Methods}

\subsection{Study design and participants}

This cross-sectional study was carried out among the school going adolescent girls in the field practice area of our institution, Madurai. All the adolescent girls aged above 10 years and attained menarche atleast one year prior to the data collection period were taken up for the study. The study was conducted for a period of three months between December 2018 and February 2019.

\subsection{Sample size and sampling method}

Based on the previous published literature, the prevalence of menstrual problems was found to be $87.7 \%$ in Tamil Nadu. ${ }^{2}$ At $95 \%$ confidence limits and $5 \%$ relative precision, the sample size was calculated as 215.5. Accounting $10 \%$ for nonresponse, the final sample size was arrived at 236 and was rounded off to 240 .

A two-stage sampling technique was employed in this study. The field practice area of our institution consists of 13 high and higher secondary schools (both government and private). Each school was individually approached for obtaining permission for the study. Out of the 13 schools, five schools consented for the study. The sample size was divided among these five schools by probability proportion to size sampling. Within each school, the list of girls studying from classes VI to XII were obtained from the register. The required number of participants from each school was selected from this list, using simple random sampling based on computer generated random numbers.

\subsection{Ethical approval and informed consent}

Approval was obtained from the Institution Ethics Committee prior to the commencement of the study. Each participant and their parents were explained in detail about the study and informed consent was obtained from the parents and the participant prior to the commencement of data collection.

\subsection{Data collection and analysis}

A structured interview schedule was used to obtain information regarding the demographic details, particulars regarding menstrual health problems and hygiene practices followed during menstruation. The data was entered and analyzed using SPSS ver.20 software. The prevalence of menstrual problems and menstrual hygiene practices were presented in percentages. The association between the menstrual problems/ hygiene practices and various risk factors were evaluated using chi square test. A p value $<0.05$ was considered statistically significant.

\subsection{Operational definition}

The socioeconomic status of the study participants was calculated based on per capita income using Modified Prasad's classification. A scoring system was used to classify the menstrual hygiene as adequate or inadequate, based on the participant's practices regarding washing the genitalia, taking bath during menstruation, type of absorbent used, frequency of change of absorbent and hygienic disposal of the absorbent material. For each of these items, a score of ' 1 ' indicates adequate practice and a score of ' 0 ' indicates poor practice. A score of ' 5 ' indicates adequate menstrual hygiene and any score below ' 5 ' indicates poor menstrual hygiene.

\section{Results}

The present study was conducted among 244 adolescent girls between the age group of 13 and 19 years. The mean age of the study participants was $14.5 \pm 0.9$ years. Majority of the participants belonged to the age group of 13-15 years $(87.7 \%)$ and belonged to lower middle socioeconomic status $(50.4 \%)$. Most of the participants hailed from nuclear family (85.7\%). (Table 1)

Table 1: Background characteristics

\begin{tabular}{llcc}
\hline $\begin{array}{l}\text { S. } \\
\text { No }\end{array}$ & Characteristics & $\begin{array}{c}\text { Frequency } \\
(\mathbf{N = 2 4 4 )}\end{array}$ & $\begin{array}{c}\text { Percentage } \\
(\boldsymbol{\%})\end{array}$ \\
1 & Age (in years) & 214 & 87.7 \\
& $13-15$ & 30 & 12.3 \\
2 & $16-19$ & & \\
& Place of residence & 35 & 14.3 \\
& Rural & 205 & 85.7 \\
3 & Urban & & \\
& Socioeconomic & & \\
& status & 20 & 8.2 \\
& Upper & 78 & 32 \\
& Upper middle & 123 & 50.4 \\
& Lower middle & 7 & 2.9 \\
& Upper lower & 16 & 6.6 \\
& Lower & & \\
4 & Type of family & 209 & 85.7 \\
& Nuclear & 35 & 14.3 \\
\hline
\end{tabular}


The present study showed a high prevalence for dysmenorrhea $(75.4 \%)$ out of which $42.9 \%$ had moderate pain. Among the other menstrual problems, irregular menstrual cycles was observed in $26.2 \%$ of the participants, while menorrhagia was seen in $34 \%$. The predominant systemic symptom associated with menstrual problems was backpain $(58.6 \%)$. (Table 2 )

Table 2: Prevalence of menstrual problems

\begin{tabular}{llcc}
\hline S. & Problem & $\begin{array}{c}\text { Frequency } \\
\text { No. }\end{array}$ & $\begin{array}{c}\text { Percentage* } \\
(\mathbf{\%})\end{array}$ \\
1 & Irregular menstrual cycles & 64 & 26.2 \\
2 & Menorrhagia & 83 & 34.0 \\
3 & Scanty bleeding & 96 & 39.3 \\
4 & Frequent cycles & 58 & 23.8 \\
5 & Dysmenorrhea & 184 & 75.4 \\
6 & Severity of pain (n=184) & & \\
& Mild & 69 & 37.5 \\
& Moderate & 79 & 42.9 \\
& Severe & 36 & 19.6 \\
7 & Other systemic symptoms & & \\
& Back pain & 143 & 58.6 \\
& Vomiting & 9 & 3.7 \\
& Irritability & 26 & 10.7 \\
& Bloating & 136 & 55.7 \\
\hline
\end{tabular}

*percentage will not totalto 100

The menstrual hygiene practices were assessed and tabulated. About $72.1 \%$ of the participants reported that they consume over the counter medicines for menstrual problems. Majority of the participants (92.2\%) use sanitary napkins as absorbent materials, and change these napkins every four hours $(94.7 \%)$. Only $22.1 \%$ of the participants wash their genitalia with soap and water during menstruation. About $10.2 \%$ of the participants do not take bath during menstruation. With regards to the disposal of sanitary napkins, $11.1 \%$ throw used napkins in open area, while $3.7 \%$ dispose in the dustbin without wrapping the napkins in paper. (Table 3)

Majority of the participants reported adequate access to change the sanitary napkins in schools (52.9\%). Similarly, $55.3 \%$ of the participants reported adequate access to hygienic disposal of the absorbent material in schools.(Figure 1) In this study, majority of the participants had adequate menstrual hygiene $(64.8 \%)$ while $35.2 \%$ of the participants had poor menstrual hygiene. (Table 4)

Among the various factors which affect the menstrual hygiene, it was observed that washing genitalia with plain water was more prevalent among the age group $>15$ years $(80 \%)$ compared to the age group of $<15$ years $(77.1 \%)$. The observed difference was statistically significant. Similarly, participants $>15$ years of age were increasingly practicing poor hygiene (no bath during menstruation) (23.3\%) compared to participants $<15$ years of age $(8.4 \%)$. The observed difference was statistically significant $(\mathrm{p}<0.05)$. (Table 5)
Table 3: Menstrual hygiene practices

\begin{tabular}{|c|c|c|c|}
\hline S. No. & Practices & $\begin{array}{c}\text { Frequency } \\
(\mathrm{N}=\mathbf{2 4 4})\end{array}$ & $\begin{array}{c}\text { Percentage } \\
(\%)\end{array}$ \\
\hline \multirow[t]{4}{*}{1} & \multicolumn{3}{|c|}{ Self-care for menstrual problems } \\
\hline & Rest & 67 & 27.5 \\
\hline & $\begin{array}{l}\text { Over the counter } \\
\text { medicines }\end{array}$ & 176 & 72.1 \\
\hline & $\begin{array}{l}\text { Hot water } \\
\text { compression }\end{array}$ & 1 & 0.4 \\
\hline \multirow[t]{4}{*}{2} & \multicolumn{3}{|c|}{ Type of absorbent used } \\
\hline & Sanitary napkin & 225 & 92.2 \\
\hline & Cloth & 4 & 1.2 \\
\hline & Others & 1 & 0.4 \\
\hline \multirow[t]{3}{*}{3} & \multicolumn{3}{|c|}{ Frequency of change of absorbent } \\
\hline & $<4$ hours & 231 & 94.7 \\
\hline & $>4$ hours & 13 & 5.3 \\
\hline \multirow[t]{4}{*}{4} & \multicolumn{3}{|c|}{ Washing genitalia during menstruation } \\
\hline & With plain water & 189 & 77.5 \\
\hline & $\begin{array}{l}\text { With soap and } \\
\text { water }\end{array}$ & 54 & 22.1 \\
\hline & $\begin{array}{l}\text { Hygiene wash } \\
\text { (with antiseptic/ } \\
\text { disinfectant) }\end{array}$ & 1 & 0.4 \\
\hline \multirow[t]{4}{*}{5} & \multicolumn{3}{|c|}{ Taking bath during menstruation } \\
\hline & Once a day & 99 & 40.6 \\
\hline & Twice a day & 120 & 49.2 \\
\hline & No bath & 25 & 10.2 \\
\hline \multirow[t]{4}{*}{6} & \multicolumn{3}{|l|}{ Method of disposal } \\
\hline & Wrap in a paper & 208 & 85.2 \\
\hline & Dispose in dustbin & 9 & 3.7 \\
\hline & $\begin{array}{l}\text { Throw in open } \\
\text { area }\end{array}$ & 27 & 11.1 \\
\hline
\end{tabular}

Table 4: Menstrual hygiene scoring and prevalence

\begin{tabular}{lccc}
\hline S. No. & Problem & Frequency & Percentage \\
1 & Adequate & 158 & 64.8 \\
2 & Poor & 86 & 35.2 \\
\hline
\end{tabular}

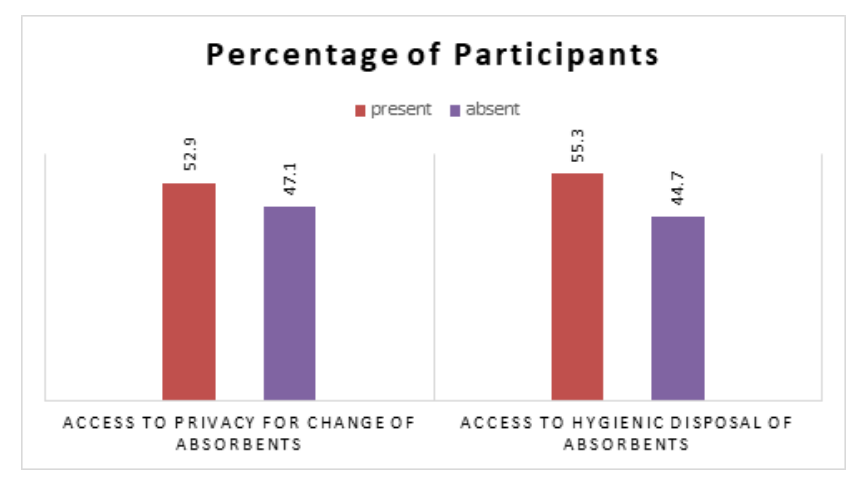

Fig. 1: Access to hygienic menstrual practices 
Table 5: Factors affecting menstrual hygiene

\begin{tabular}{|c|c|c|c|c|c|c|}
\hline & \multicolumn{4}{|c|}{ Menstrual hygiene component } & \multirow[t]{3}{*}{ Chi sq } & \multirow[t]{2}{*}{$P$ value } \\
\hline \multirow{2}{*}{\multicolumn{6}{|c|}{$\begin{array}{l}\text { Washing genitalia } \\
\text { Soap and water }\end{array}$}} & \\
\hline & & & & & & \\
\hline$<15$ & 214 & $165(77.1)$ & $49(22.9)$ & $0(0)$ & \multirow{2}{*}{7.6} & \multirow{2}{*}{0.022} \\
\hline$>15$ & 30 & $24(80)$ & $5(16.7)$ & $1(3.3)$ & & \\
\hline \multicolumn{7}{|c|}{ Bathing during menstruation } \\
\hline & & Once a day & Twice a day & No bath & \multirow{3}{*}{7.6} & \multirow{3}{*}{0.023} \\
\hline$<15$ & 214 & $86(40.2)$ & $110(51.4)$ & $18(8.4)$ & & \\
\hline$>15$ & 30 & $13(43.3)$ & $10(33.3)$ & $7(23.3)$ & & \\
\hline
\end{tabular}

In this study, socioeconomic status was observed to be a significant predictor for the type of absorbent material used. While majority of the participants in the upper classes use sanitary napkins, cloth and other materials were used predominantly by the lower and upper lower classes. The observed difference was found to be statistically significant $(\mathrm{p}<0.002)$. (Table 6)

The place of residence had a significant impact on the health seeking pattern of the study participants. It was observed that participants in the rural area had increased use of over the counter medicines $(91.4 \%)$ compared to their urban counterparts $(68.9 \%)$. The observed difference was statistically significant $(\mathrm{p}<0.023)$. (Table 7)

\section{Discussion}

Hygiene is an essential component of health and well being and menstrual hygiene plays a key role in various uro-gynecological, sexually transmitted and reproductive tract infections. It is always advantageous to impart the knowledge on menstrual hygiene early during the adolescence as the adolescent age group is ideal for inculcating healthy habits backed with adequate scientific evidence. The present study evaluated the prevalence of menstrual problems and menstrual hygiene. The mean age of the participants was 14.5 years. In a study done by Ravi $\mathrm{R}$ et al., the mean age of the participants was 14.7 years, similar to our study. ${ }^{2}$ In another study done by Omidvar S et al., the mean age of the participants was 15.6 years, slightly higher than the present study. ${ }^{4}$

In this study, dysmenorrhea was the most predominant menstrual problem (75.4\%) followed by menorrhagia (34\%). Moreover, backpain was also highly prevalent $(58.6 \%)$ among the study participants. The above findings were similar to the studies published in South India, with dysmenorrhea being the predominant menstrual problem in a study done by Ravi R (72.6\%), Rafique N (89.7\%), Omivdar S et al $(66.8 \%){ }^{2,4,5}$ Although menstruation is a normal phenomenon, the burden of menstrual problems is increasing among the adolescents, as observed in the present study and published literature. The increasing prevalence of menstrual problems significantly impacts the physical and psychosocial well being of these adolescents, causing poor social well being and interpersonal relationships with $78.5 \%$ reported school absenteeism and poor participation in social and sports activities as high as $51.1 \% .^{6}$

The menstrual hygiene in the present study was assessed based on four cardinal issues namely taking bath during menstruation, frequency of change of the absorbent material, hygienic disposal of the absorbent material and washing genitalia with soap and water. In the present study, $64.8 \%$ of the participants had adequate menstrual hygiene. About $22.1 \%$ of the participants wash their genitalia with soap and water about $10.2 \%$ do not take bath during menstruation. Age was a significant risk factor for certain menstrual hygiene practices namely washing genitalia during menstruation and taking bath during menstruation, for which age $>15$ years showed poor hygiene practices compared to the younger age groups. The observed difference was statistically significant $(\mathrm{p}<0.05)$. In a study done by Das $\mathrm{P}$ et al, only $14.8 \%$ practice adequate hygiene in terms of taking bath during menstruation and $84.7 \%$ of the participants had full body bath including wash of genitalia. ${ }^{7}$

In terms of menstrual practices, the present study observed that majority of the participants used sanitary napkins $(92.2 \%)$. It was observed that participants in the lower socioeconomic status used cloth and other materials $(18.8 \%)$ compared to those in the upper socioeconomic status $(0 \%)$. The observed difference was statistically significant $(\mathrm{p}<0.001)$ Furthermore, $52.9 \%$ of the participants had access to change these napkins in privacy and $55.3 \%$ had access to hygienic disposal of the absorbent materials. In a case control study done by Das P et al, $48.7 \%$ of the participants were found to be using sanitary napkins, which was lower than our study estimates. In addition, about $24.1 \%$ of the participants had private access to change of pads. $^{7}$

The health seeking behavior for the menstrual problems was evaluated in the present study and it was observed that $72.1 \%$ of the participants consumed over-the-counter medicines for the menstrual problems while $27.5 \%$ took physical rest. The prevalence of over-the-counter medicine consumption was higher among the participants from rural area $(91.4 \%)$ compared to the urban areas $(68.9 \%)$. The 
Table 6: Role of socioeconomic status in menstrual practices

\begin{tabular}{|c|c|c|c|c|c|c|}
\hline \multirow{2}{*}{$\begin{array}{l}\text { Socioeconomic } \\
\text { status }\end{array}$} & & \multicolumn{3}{|c|}{ Type of absorbent used } & \multirow{2}{*}{ Chi sq } & \multirow{2}{*}{$P$ value } \\
\hline & & Sanitary napkin & Cloth & Others & & \\
\hline Upper & 20 & $20(100)$ & $0(0)$ & $0(0)$ & & \\
\hline Upper middle & 78 & $72(92.3)$ & $2(2.6)$ & $4(5.1)$ & & \\
\hline Lower middle & 123 & $116(94.3)$ & $2(1.6)$ & $5(4.1)$ & 24.1 & 0.002 \\
\hline Upper lower & 7 & $4(57.1)$ & $0(0)$ & $3(42.9)$ & & \\
\hline Lower & 16 & $13(81.3)$ & $0(0)$ & $3(18.8)$ & & \\
\hline
\end{tabular}

Table 7: Role of residence in choice of treatment

\begin{tabular}{lccccc}
\hline $\begin{array}{l}\text { Place of } \\
\text { residence }\end{array}$ & Rest & $\begin{array}{c}\text { Health seeking behavior } \\
\text { Over the counter } \\
\text { medicines }\end{array}$ & $\begin{array}{c}\text { Hot water } \\
\text { compression }\end{array}$ & Chi sq & P value \\
Rural & 35 & $3(8.6)$ & $32(91.4)$ & $0(0)$ & 7.6 \\
Urban & 209 & $64(30.6)$ & $144(68.9)$ & $1(0.5)$ & 0.023 \\
\hline
\end{tabular}

observed difference was statistically significant $(\mathrm{p}<0.05)$. In a study done by Acheampong $\mathrm{K}$ et al, $34.6 \%$ of the participants with dysmenorrhea practiced self- medications, which was lower than our study estimates. ${ }^{8}$ Very few studies have documented this observation. A study done by Selvaraj K et al observed that $9.5 \%$ of the study population consumed over-the-counter medications for dysmenorrhea, which is lower than the present study estimates. ${ }^{9}$

\section{Conclusion}

Menstrual hygiene is an essential component of menstrual health. The present study has shown a high prevalence of dysmenorrhea, for which majority of the participants practice over-the-counter medications. Considering the significant correlation between socioeconomic status and the choice of absorbent used, there is an increasing necessity to make quality sanitary napkins available and affordable for the lower socioeconomic groups. This can be achieved with the help of public infrastructure and collaborating with cooperative societies. This study has emphasized on the need for creating awareness during the early adolescent age in order to inculcate hygienic menstrual practices. Moreover, there is a growing need to incorporate adolescent specific health services in the public health system to reach out to the adolescent girls in order to prevent hazardous practices like over-the-counter medication use.

\section{Conflict of Interest}

Nil.

\section{Acknowledgements}

The study is a part of Madurai Obstetrics and Gynecological Society (MOGS) and the authors are thankful to MOGS for the opportunity.

\section{References}

1. World Health Organization. Adolescent Pregnancy. Key Facts. [Internet] . Available from: https://www.who.int/news-room/factsheets/detail/adolescent-pregnancy.

2. Ravi R, Shah P, Palani G, Edward S, Sathiyasekaran BWC. Prevalence of Menstrual Problems among Adolescent School Girls in Rural Tamil Nadu. J Pediatr Adolesc Gynecol. 2016;29(6):571-6. doi:10.1016/j.jpag.2015.10.016

3. Patil SS, Deshpande TN, Gharai SB, Patil SR, Durgawale PM. Menstrual hygiene among adolescent girls - A study from urban slum area. J Fam Med Prim Care. 2018;7(6):1439-45. do1:10.4103/]fmpc.jtmpc_80_18.

4. Bakhtiari A, Omidvar S, Amiri FN, Begum K. A study on menstruation of Indian adolescent girls in an urban area of South India. J Fam Med Prim Care. 2018;7(4):698-702. doi:10.4103/jfmpc.jfmpc_258_17.

5. Rafique N, Al-Sheikh MH. Prevalence of menstrual problems and their association with psychological stress in young female students studying health sciences. Saudi Med J. 2018;39(1):67-73. do1:10.15537/sm].2018.1.21438.

6. Ravi R, Shah PB, Edward S, Gopal P, Sathiyasekaran BWC. Social impact of menstrual problems among adolescent school girls in rural Tamil Nadu. Int J Adolesc Med Health. 2018;30(5). doi:10.1515/1jamh2016-0088.

7. Das P, Baker KK, Dutta A, Swain T, Sahoo S, Das BS. Menstrual hygiene practices, WASH access and the risk of urogenital infection in women from Odisha. PLoS one. 2015;10(6):e0130777.

8. Acheampong K, Baffour-Awuah D, Ganu D, Appiah S, Pan X, Kaminga A, et al. Prevalence and Predictors of Dysmenorrhea, Its Effect, and Coping Mechanisms among Adolescents in Shai Osudoku District, Ghana. Obstet Gynecol Int. 2019;2019:1-7. d01:10. $155 / 2010 / 5834159$

9. Kumar GS, Selvaraj K, Ramalingam A. Prevalence of self-medication practices and its associated factors in Urban Puducherry, India. Perspect Clin Res. 2014;5(1):32-6. do1:10.4103/2224-3485. 24569

\section{Author biography}

Lakshmi Subburaj, Consultant, Gynecologist and Laparoscopic Surgeon

K Rajkumar, Consultant Surgical Gastroenterologist

Cite this article: Subburaj L, Rajkumar K. Menstrual hygiene and their predictors among school going adolescent girls in Tamil Nadu. Indian J Obstet Gynecol Res 2021;8(2):250-254. 